\title{
Photoisomerization of Provitamin D in Hydrogel Matrix
}

\author{
I. P. Terenetskaya ${ }^{1}$, Yu. M. Samchenko ${ }^{2}$, T. N. Orlova ${ }^{1}$, N. A. Pasmurceva ${ }^{2}$, P. S. Kapinos ${ }^{1}$, \\ I. E. Boldeskul', Z. R. Ulberg ${ }^{2}$ \\ ${ }^{1}$ Institute of Physics, NAS Ukraine, Prospekt Nauki, Kyiv, Ukraine \\ ${ }^{2}$ Ovcharenko Institute of Biocolloid Chemistry, NAS Ukraine, Acad. Vernadsky Blvd., \\ Kyiv, Ukraine \\ Email: tereneti@ukr.net
}

Received April 26, 2013; revised June 12, 2013; accepted June 19, 2013

Copyright (C) 2013 I. P. Terenetskaya et al. This is an open access article distributed under the Creative Commons Attribution License, which permits unrestricted use, distribution, and reproduction in any medium, provided the original work is properly cited.

\begin{abstract}
Study of interfacial processes between incorporated biomolecules and hydrogel matrix is of primary importance for the application of synthetic hydrogels as biomaterials, sensors, etc. Their hydrophilic nature, however, hampers fat-soluble biomolecule incorporation. We synthesized N-isopropylacrylamide (NIPAAm)- and Acrylamide (AA)-based hydrogels with hydrophilic-lipophilic balance that under specific conditions permit homogeneous incorporation of provitamins $\mathrm{D}_{3}$ (7-dehydrocholesterol) and $\mathrm{D}_{2}$ (ergosterol) dissolved in ethanol. Similarity of provitamins D photoisomerization in such hydrogel matrices with the photoreaction in ethanol shows great potential of the novel material for personal dosimetry of biologically active 'antirachitic' UV radiation.
\end{abstract}

Keywords: Hydrogels; Provitamin D Photoisomerization; UV Biodosimeter

\section{Introduction}

Influence of a reaction medium on the photochemistry of biologic molecules is of great current interest. Photoisomerization of provitamin $\mathrm{D}$, in particular, as the initial step of natural synthesis of vitamin D upon sunlight, has been studied in various media [1-5]. For the most part, interaction of provitamin $\mathrm{D}$ molecules with microenvironment affects both the absorption spectrum and the photoconversions. In view of great current interest to the application of natural, synthetic, and biohybrid hydrophilic polymers as biomaterials, we have studied photoisomerization of provitamin $\mathrm{D}_{3}$ (7-dehydrochelosterol) and provitamin $\mathrm{D}_{2}$ (ergosterol) in a hydrogel matrix.

Hydrogels, the novel materials, insoluble crosslinked hydrophilic polymers that swell in aqueous medium, came in the focus of researchers' attention lately [6]. Hydrogel water content may vary from $10 \%$ to thousands of times of the weight of the dry polymer network [7]. Due to their high biocompatibility and the ability to incorporate drugs into their composition, hydrogels are widely used in medicine [8], pharmacology and biology to develop various biomaterials: implants, soft contact lenses, wound coatings, cell carriers, drug delivery systems (heparin delivery, for instance) [9], etc.

Thermo-sensitive hydrogel polymeric systems are be- ing widely studied and appear promising for medical application, first of all, for the design of targeted drug delivery and their controlled release. They are characterized by the presence of upper and/or lower critical solution temperatures (UCST and/or LCST) at which phase transition between swollen and collapsed state occurs in the system. The phase transition in polymeric hydrogels and solutions based on NIPAAm is governed by the transition between two conformation structures of the polymer side groups [10]. LCST of NIPAAm-based polymer systems is in the range of $32^{\circ} \mathrm{C}-34^{\circ} \mathrm{C}$ and slightly differs from the temperature of a human body. Nevertheless, due to the fact that the phase transition temperature of thermo-sensitive systems depends on hydrophilic-lipophilic balance, it can be shifted to any direction by copolymerization of NIPAAm with either hydrophilic or hydrophobic monomers [11].

Diphilic nature of NIPAAm-based hydrogels and that of hydrogels based on hydrophilic and hydrophobic monomers ensures their affinity not only to water and water solutions, but to organic solvents as well. Their swelling degree in such solvents reaches even higher values within certain temperature and concentration intervals. The present paper deals with the synthesis of NIPAAm- and AA-based copolymer hydrogels and their ability to be saturated with ethanol solutions of provita- 
mins $\mathrm{D}_{3}$ and $\mathrm{D}_{2}$. Effect of the reaction media on the photochemistry of the above mentioned biomolecules is studied versus the photoreaction in the ethanol solution.

\section{Materials and Methods}

N-isopropylacrylamide (NIPAAm) (Sigma-Aldrich, 97\%) was recrystallized from $n$-hexane and dried under vacuum. Acrylamide (AAm) (Merck, 99.9\%), N,N'-methylenebisacrylamide (MBA) (Merck, 98\%), ammonium persulfate (PSA) (Sigma, 98\%) and N,N,N',N'-tetramethylendiamine (TMED) (Merck, 99\%), were used as received without preliminary purification.

Hydrogel matrices for further incorporation of provitamins D were prepared as follows: for 2 minutes, argon passed through the solution containing $1.95 \mathrm{~g}$ of $\mathrm{N}$-isopropylacrylamide, $0.05 \mathrm{~g}$ of acrylamide, $0.01 \mathrm{~g}$ of $\mathrm{N}$, $\mathrm{N}$-methylenebisacrylamide, $0.1 \mathrm{~g}$ of ammonium persulphate and $6.0 \mathrm{ml}$ of water on ice. Then, $0.1 \mathrm{~g}$ of tetramethylenediamine was added to the solution and stirred on a magnet stirrer for 1 minute. The obtained solution was poured in between two flat parallel glass or Teflon plates separated by $1 \mathrm{~mm}$-thick spacers. In one hour, when polymerization was completed, the plates were separated and the obtained hydrogel films were taken out and washed to remove un-reacted monomer residues at $40^{\circ} \mathrm{C}$ during 120 hours with daily water change.

Swelling degree $Q$ in grams of a solvent per gram of a dry polymer was determined by the weight method by the formula: $Q=\frac{m_{t}-m_{0}}{m_{0}}$, where $m_{0}$ is a mass a dry xerogel, $m_{t}$ - mass of a hydrogel at the time moment $t$.

The hydrogel saturation with provitamin D was accomplished as follows. Synthesized hydrogel matrices were dried at $30^{\circ} \mathrm{C}-40^{\circ} \mathrm{C}$, then during 24 hours they were saturated with provitamin $\mathrm{D}$ solution at about $5^{\circ} \mathrm{C}$. Ethanol solutions of provitamins D with the concentration of 0.01 mass $\%$ were prepared in advance by weighting the components. To prevent ethanol evaporation, the hydrogel matrices saturated with provitamin D were hermetically sealed within propylene envelopes.

UV irradiation of the samples was performed using low-pressure mercury lamp TUV-300 (254 nm) and luminescent lamp EL-30 (280 - $330 \mathrm{~nm})$. Spectral irradiance of the UV lamps at the sample distance $7 \mathrm{~cm}$ was measured with calibrated spectrometer EPP2000C-UV + VIS (StellarNet, Inc). The photoreaction course was followed by UV absorption spectroscopy, i.e. absorption spectra of the samples were recorded by PerkinElmer Lambda 25 UV/VIS spectrophotometer within 250 - 330 $\mathrm{nm}$ before and after fixed UV exposures. Then, for the concentration analysis the spectra were processed using specially developed computer software [12]. Simultaneously, solutions of provitamins D in ethanol were irradi- ated under the same conditions.

\section{Results and Discussion}

In order to find optimal conditions of incorporating fatsoluble provitamin D in synthetic hydrogels of high biocompability caused mainly by their high water content, we carried out their synthesis using monomers of various natures. The hydrogels were obtained basing on hydrophilic acrylamide, hydrophobic acrylonitrile and acrylic acid useful in medicine for endoprosthetics, anti-burn coatings, targeted medication delivery and their prolonged release.

The traditional technique described, for instance in [11], was used in the hydrogel synthesis. The hydrogels obtained had satisfactory mechanical characteristics, but they did not swell, rather collapse, in ethanol and hexane, traditionally good solvents for provitamin D. Therefore, the hydrogel matrices mentioned above can not be used to incorporate provitamins.

It appeared that co-polymer hydrogels containing NIPA diphile chains are compatible with ethanol. Synthesis and properties of the above-mentioned hydrogel systems, as well as those of ferrogels based on them, are described in detail in [13], while the present work deals with the development of the techniques of optimizing provitamin D incorporation in the hydrogels .

In order to find optimal conditions of provitamin D incorporation in the synthesized hydrogels their swelling has been studied in distilled water and ethanol. The corresponding kinetic curves of swelling in distilled water and in ethanol at $6^{\circ} \mathrm{C}$ and at $40^{\circ} \mathrm{C}$ are given in Figure 1 .

On swelling in water, thermo-sensitive NIPA and AA copolymer-based hydrogels behave traditionally, and sharp decrease of their highest swelling degree (approximately 8 times) is observed with temperature growth due to NIPA transition to hydrophobic modification. At the same time, swelling in ethanol obeys the opposite dependence, i.e. swelling degree increases slightly with temperature growth and achieves the maximum value

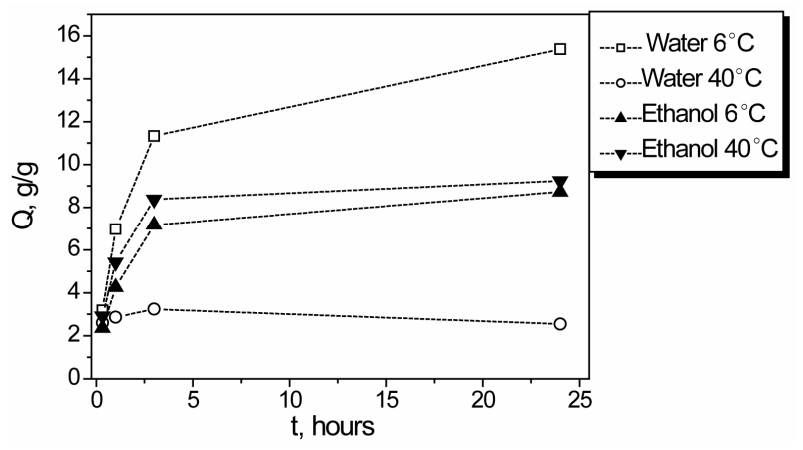

Figure 1. Kinetics of synthesized hydrogel swelling in water and in ethanol at $6^{\circ} \mathrm{C}$ and $40^{\circ} \mathrm{C}$. 
$\sim 8 \mathrm{~g} / \mathrm{g}$ in 3 hours. Contrary to conventional hydrogels, homopolyacrylamide gel in particular, the synthesized hydrogels remain optically transparent and thus can be used as matrices for water-insoluble provitamins D.

The described behavior of NIPA-based hydrogels can be explained by some peculiarities of their chemical structure, i.e. by simultaneous presence of hydrophilic amide group $(\mathrm{CONH})$ well water-solvated and hydrophobic isopropyl group $\mathrm{CH}$ (CH3), solvated by ethanol (Figure 2).

Photochemical characteristics of the behavior of provitamins $\mathrm{D}$ in the described hydrogel matrix based on NIPA are discussed below.

UV irradiation of initial provitamin D at room temperature is known to give rise to multicomponent photoisomer mixture due to the side photoconversions of formed previtamin $\mathrm{D}$ whose absorption band lies in the same spectral range as that of provitamin D [14]. In due course, dynamic equilibrium between the photoisomers, the so-called photostationary state (PS), is established. Previtamin D (Pre) and its trans-isomer tachysterol (T) usually dominate in the photostationary state, and the Pre/ $\mathbf{T}$ ratio is strongly dependent on the irradiation wavelength. Irradiation at $254 \mathrm{~nm}$ ensures high yield of tachysterol, and its amount decreases as the initiation radiation wavelength becomes longer [14]. Such difference in the photoreaction kinetics is reflected by different transformation of the initial spectrum in the process of UV irradiation. Upon irradiation at $254 \mathrm{~nm}$ the initial insignificant decrease of absorbance caused by the formation of previtamin $\mathrm{D}$, is replaced by its appreciable increase caused by tachysterol accumulation (Figure 3). During further UV irradiation, the absorbance is gradually reduced due to irreversible photodegradation (not shown).

Different picture is observed under long-wave UV irradiation by EL-30 lamp (Figure 4): the absorbance gradually decreases around maximum, at $280 \mathrm{~nm}$, because absorption coefficient of provitamin D is higher than that of previtamin D in this spectral range. However, the absorbance increase is observed at $260 \mathrm{~nm}$ (maximum absorption of previtamin D).

Similar transformations were detected upon UV irradiation with both lamps for the hydrogel films with incorporated 7-DHC (provitamin D3). It is significant that unlike previous data on provitamin D doped polymer and porous silica films [5], the absorption spectra of both provitamins in hydrogel matrices were almost identical to the spectra in ethanol solution. With the help of specifically developed software [12], the above-mentioned fact

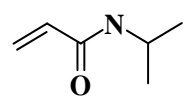

Figure 2. Structural formula of NIPA.

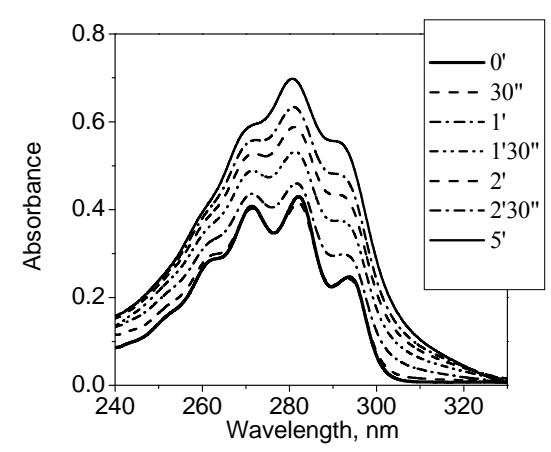

(a)

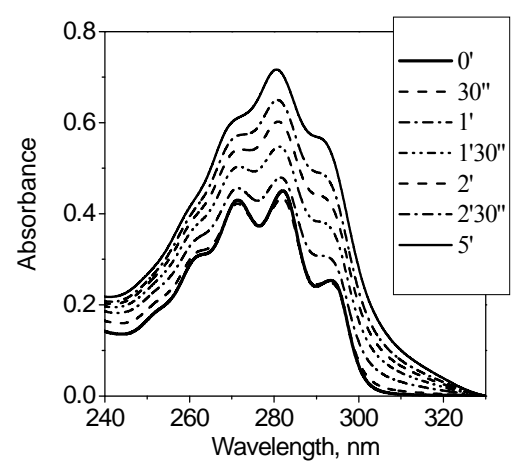

(b)

Figure 3. Transformation of ergosterol absorption spectrum in hydrogel matrix (a) and in ethanol (b) at the initial stage of UV irradiation with the TUV-300 lamp.

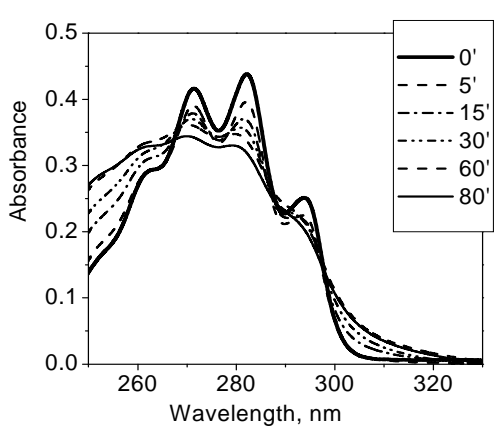

(a)

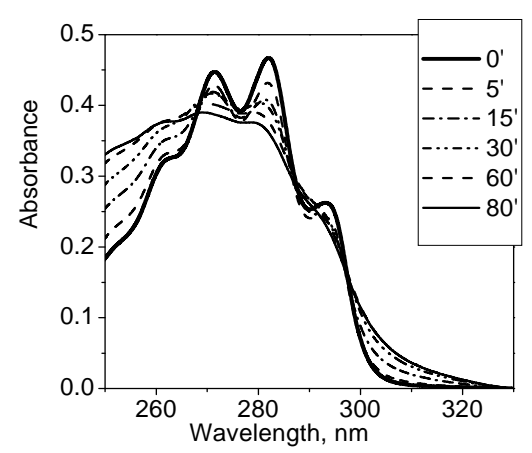

(b)

Figure 4. Transformation of ergosterol absorption spectrum in hydrogel matrix (a) and in ethanol (b) upon UV irradiation with the EL-30 lamp. 
allowed processing the spectra for concentration analysis based on the known photoisomer individual spectra in the solution [15].

Comparison of concentration kinetics of provitamin D photoisomerization in hydrogel matrix and in ethanol demonstrates their close similarity upon irradiation with both UV lamps (Figure 5). By this is meant that the interaction between incorporated molecules of provitamin $\mathrm{D}$ and the hydrogel matrix appears weak enabling us to suggest that the molecules are not adsorbed but stay "swimming" in ethanol inside the hydrogel micro-cavities.

This situation differs from the other heterogeneous media where restrictions of molecular geometry caused by interfacial interactions significantly affect the conformational and dynamic behavior of electronically excited molecules, and, as a result, change the photoreaction pathways and product distribution. This is especially true in regard to the flexible molecule of previtamin D whose multiple photoconversions are best rationalized on the base of the principle of ground state conformational control [16]. Pronounced changes revealed in the kinetics of provitamin D photoisomerization in some heterogeneous media [17-19] were attributed to the displacement of previtamin $\mathrm{D}$ conformational equilibrium by the restriction of its intramolecular rotation around C5-C6 sin-

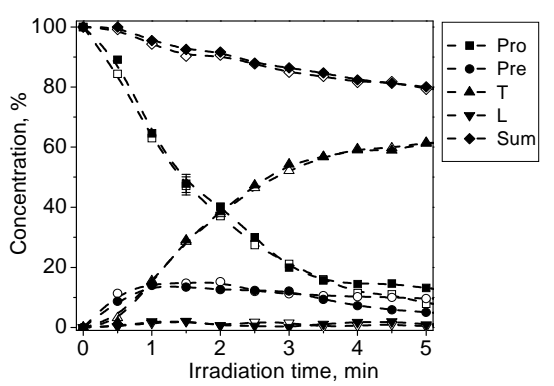

(a)

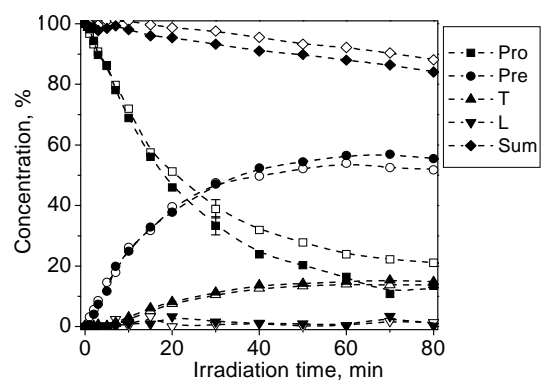

(b)

Figure 5. Concentration kinetics of ergosterol photoisomerization in hydrogel matrix (solid symbols) and in ethanol (open symbols) upon irradiation with TUV-300 (a) and EL-30 (b) lamps: Pro-provitamin D, Pre-previtamin D, T-tachysterol, L-lumisterol, Sum-their summary concentration.

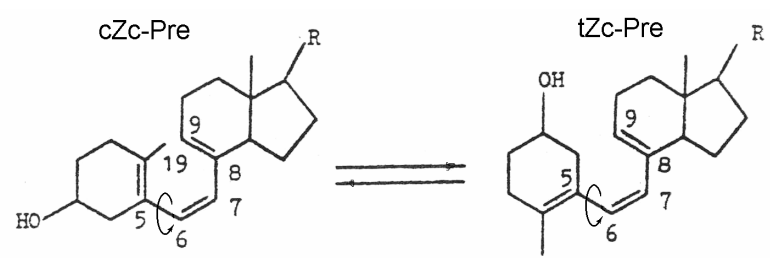

Figure 6. Two planar conformations of previtamin D molecule.

gle bond. As it was shown earlier [1], cis-trans isomerization of previtamin $D$ is especially sensitive to the ratio between its planar $c Z c$-Pre and $t Z c$-Pre conformers (Figure 6), and shift of conformation equilibrium in favor of the $c Z c$-Pre due to adsorption of previtamin $\mathrm{D}$ at the silica gel surface significantly inhibited its cis-trans isomerization [1].

\section{Conclusions}

Close resemblance of provitamin D photoisomerization in hydrogel matrices to the photoreaction in ethanol gives grounds to assume that the molecules of provitamin D inside hydrogel matrix are not distorted by interfacial interactions. This offers a clue for personal dosimetry of "antirachitic" UV radiation [20] similar to an original method based on an in vitro model of vitamin D synthesis (the so-called "D-dosimeter") that was introduced for the UV dosimetry in situ [21].

Great potential of the new material for personal monitoring of the vitamin-D-synthetic capacity of sunlight is motivated by the crucial importance of vitamin $D$ for human health [22].

\section{REFERENCES}

[1] I. P. Terenetskaya, O. G. Dmitrenko and A. M. Eremenko, "Conformational Control in Previtamin D Photochemistry by Heterogeneous Reaction Media," Research on Chemical Intermediates, Vol. 21, No. 6, 1995, pp. 653-664. doi:10.1163/156856795X00495

[2] O. G. Dmitrenko, I. P. Terenetskaya and W. Reischl, "Sol- vent Effect on Previtamin D Conformational Equilibrium and Its Photoreactions," Journal of Photochemistry and Photobiology A: Chemistry, Vol. 104, No. 1-3, 1997, pp. 113-117. doi:10.1016/S1010-6030(97)04597-8

[3] I. Gvozdovskyy, T. Orlova and I. Terenetskaya "Features of Previtamin D cis-trans Isomerization in the Nematic LC Matrices: Orientation and Cholesteric Order Effects," Molecular Crystals and Liquid Crystals, Vol. 434, No. 1, 2005, pp. 325-332. doi:10.1080/15421400590955361

[4] T. N. Orlova and I. P. Terenetskaya, "Specific Features of Photoisomerization of Provitamin $\mathrm{D}_{3}$ in a Cholesteric Liquid Crystal," Optics and Spectroscopy, Vol. 104, 2010, pp. 584-589. doi:10.1134/S0030400X10040156

[5] T. N. Orlova, I. P. Terenetskaya, A. M. Eremenko and N. I. Surovtseva, "Provitamin D Doped Silica and Polymeric 
Films: New Materials for UV Biosensor," Materials Sciences and Applications, Vol. 1, 2010, pp. 267-271.

[6] O. Wichterle and D. Lim, "Hydrophilic Gels for Biological Use," Nature, Vol.185, 1960, pp. 117-118. doi:10.1038/185117a0

[7] T. Tanaka, "Gels, Encyclopedia of Polymer Science and Engineering,” Wiley, New York, 1987, pp. 514-520.

[8] I. Galaev and B. Mattiasson, "'Smart' Polymers and What They Could Do in Biotechnology and Medicine," Trends in Biotechnology, Vol. 17, No. 8, 1999, pp. 336-340. doi:10.1016/S0167-7799(99)01345-1

[9] C. S. Brazel and N. A. Peppas, "Pulsatile Local Delivery of Thrombolytic and Antithrombotic Agents Using Poly(N-isopropyl acrylamideo-methacrylic acid) Hydrogels," Journal of Controlled Release, Vol. 39, No. 1, 1996, pp. 57-64. doi:10.1016/0168-3659(95)00134-4

[10] N. A. Plate, T. L. Lebedeva and L. I. Valuev, "Lower Critical Solution Temperature in Aqueous Solutions of Nalkyl-substituted Polyacrylamides," Polymer Journal, Vol. 31, 1999, pp. 21-27. doi:10.1295/polymj.31.21

[11] Yu. Samchenko, Z. Ulberg and O. Korotych "Multi-purpose Smart Hydrogel Systems," Advances in Colloid and Interface Science, Vol. 168, No. 1-2, 2011, pp. 247-262. doi:10.1016/j.cis.2011.06.005

[12] I. Terenetskaya, "Spectral Monitoring of Biologically Active Solar UVB Radiation Using an in Vitro Model of Vitamin D Synthesis," Talanta, Vol. 53, No. 1, 2000, pp. 195-203. doi:10.1016/S0039-9140(00)00459-8

[13] O. Korotych, Yu. Samchenko, I. Boldeskul, Z. Ulberg, N. Zholobak and L. Sukhodub, "N-IsopropylacrylamideBased Fine-Dispersed Thermosensitive Ferrogels Obtained via in-Situ Technique," Materials Science and Engineering C, Vol. 33, 2013, pp. 892-900.

[14] E. Havinga, "Vitamin D, Example and Challenge," Experentia, Vol. 29, No. 10, 1973, pp. 81-93. doi:10.1007/BF01935064
[15] J. C. Sternberg, H. S. Stillo and R. H. Schwendeman, "Spectrophotometric Analysis of Multicomponent Systems Using the Least Squares Method in Matrix Form. The Ergosterol Irradiation System," Analytical Chemistry, Vol. 32, No. 1, 1960, pp. 84-90. doi:10.1021/ac60157a025

[16] H. J. C. Jacobs and E. Havinga, "Photochemistry of Vitamin D and Its Isomers and of Simple Trienes," Advances in Photochemistry, Vol. 11, 1979, pp. 305-373.

[17] I. P. Terenetskaya, O. G. Perminova and A. M. Yeremenko, "Effect of Environment on the Conformational Equilibrium and Photoconversions of Previtamin D," Journal of Molecular Structure, Vol. 267, 1992, pp. 93-98. doi:10.1016/0022-2860(92)87015-N

[18] J. K. Yamamoto and R. F. Borch, "Regulation of Cutaneous Previtamin D Photosynthesis in Man Skin: Conversion of 7-Dehydrocholesterol to Vitamin $\mathrm{D}_{3}$ in Synthetic Phospholipid Bilayers," Biochemistry, Vol. 24, No. 13, 1985, pp. 3338-3344. doi:10.1021/bi00334a039

[19] R. M. Moriarty, R. N. Schwartz, C. Lee and V. Curtis, "Formation of Vitamin $\mathrm{D}_{3}$ in Synthetic Lipid Multibilayers. A Model for Epidermal Photosynthesis," Journal of the American Chemical Society, Vol. 102, No. 12, 1980, pp. 4257-4259. doi:10.1021/ja00532a047

[20] O. N. Galkin and I. P. Terenetskaya, "Vitamin D Biodosimeter: Basic Characteristics and Prospect Applications," Journal of Photochemistry and Photobiology B: Biology, Vol. 53, 1999, pp. 12-19. doi:10.1016/S1011-1344(99)00115-3

[21] I. P. Terenetskaya, "Provitamin D Photoisomerization as Possible UVB Monitor: Kinetic Study Using Tunable Dye Laser," SPIE Proceedings, Vol. 2134B, 1994, pp. 135-140. doi:10.1117/12.180825

[22] A. W. Norman and R. Bouillon, "Vitamin D Nutrition Policy Needs a Vision for Future," Experimental Biology and Medicine, Vol. 235, 2010, pp. 1034-1045. 